TRANSACTIONS OF THE

AMERICAN MATHEMATICAL SOCIETY

Volume 360, Number 5, May 2008, Pages 2661-2680

S 0002-9947(07)04324-3

Article electronically published on December 11, 2007

\title{
INVARIANT SUBSPACES FOR BANACH SPACE OPERATORS WITH AN ANNULAR SPECTRAL SET
}

\author{
ONUR YAVUZ
}

\begin{abstract}
Consider an annulus $\Omega=\left\{z \in \mathbb{C}: r_{0}<|z|<1\right\}$ for some $0<$ $r_{0}<1$, and let $T$ be a bounded invertible linear operator on a Banach space $X$ whose spectrum contains $\partial \Omega$. Assume there exists a constant $K>0$ such that $\|p(T)\| \leq K \sup \{|p(\lambda)|:|\lambda| \leq 1\}$ and $\left\|p\left(r_{0} T^{-1}\right)\right\| \leq K \sup \{|p(\lambda)|:|\lambda| \leq$ $1\}$ for all polynomials $p$. Then there exists a nontrivial common invariant subspace for $T^{*}$ and $T^{*-1}$.
\end{abstract}

\section{INTRODUCTION}

Ambrozie and Müller $[\mathrm{AM}]$ proved that the adjoint of a polynomially bounded operator whose spectrum contains the unit circle has a nontrivial invariant subspace. The result generalizes the theorem of Brown, Chevreau, and Pearcy [BCP], which applies to Hilbert space contractions (see also [B] for an exposition of this result). In this paper, we prove an analogous result where the unit circle is replaced by the boundary of an annulus.

Let $\Omega=\left\{z \in \mathbb{C}: r_{0}<|z|<1\right\}$ for some $0<r_{0}<1$. Recall that a bounded linear operator $T$, defined on a complex Banach space $X$, is said to be polynomially bounded if there exists a constant $K>0$ such that $\|p(T)\| \leq K \sup \{|p(\lambda)|:|\lambda| \leq$ $1\}$ for all polynomials $p$, and the constant $K$ is said to be the polynomial bound of $T$.

Our main result can be formulated as follows:

Theorem A. Let $T$ be an invertible bounded linear operator on a complex Banach space $X$ whose spectrum contains $\partial \Omega$, and such that $T$ and $r_{0} T^{-1}$ are polynomially bounded. Then there exists a nontrivial common invariant subspace for $T^{*}$ and $T^{*-1}$.

The fact that each of the operators $T^{*}$ and $T^{*-1}$ has a nontrivial invariant subspace follows from $[\mathrm{AM}]$. Our contribution is that there exists a proper subspace invariant for both of them.

The main tools used in proving the main result of $[\mathrm{AM}]$ are an improved version of Zenger's theorem, Apostol's theorem, and Carleson's interpolation theorem. Sections 2 and 3 of this paper are devoted to these theorems and construction of the essential technical tools they lead to. In Section 4, we provide some estimates for the Poisson kernels on $\Omega$. As in the disk, these act as representing measures for

Received by the editors May 2, 2005 and, in revised form, April 17, 2006.

2000 Mathematics Subject Classification. Primary 47A15; Secondary 47A60.

(C) 2007 American Mathematical Society 
point evaluations of analytic functions in $\bar{\Omega}$ (see $[\mathrm{S}]$ ). In Section 5 these evaluation functionals are "factored", and this yields the desired invariant subspaces.

\section{Preliminaries}

Let us denote by $A(\bar{\Omega})$ the Banach algebra of continuous functions in $\bar{\Omega}$ which are analytic on $\Omega$ with sup norm, by $H^{\infty}(\Omega)$ the Banach algebra of bounded analytic functions on $\Omega$, and by $L(X)$ the Banach algebra of bounded linear operators on $X$.

First we would like to reduce the proof of Theorem A to the case in which $T$ admits a functional calculus with functions from $H^{\infty}(\Omega)$ and $\|h(T)\|=\|h\|_{\infty}$, for all $h \in H^{\infty}(\Omega)$.

By analogy with the case of the unit disk, we give the following definition:

Definition (Apostol set). A subset $\Lambda \subset \Omega$ is called an Apostol set if $\sup \{r \in$ $\left.\left(r_{0}, 1\right): r e^{i \theta} \in \Lambda\right\}=1$ and $\inf \left\{r \in\left(r_{0}, 1\right): r e^{i \theta} \in \Lambda\right\}=r_{0}$ for all but countably many $\theta \in(-\pi, \pi]$.

The following result follows from the original result (Lemma 2.1 in [A1]) by Apostol applied to $T$ and $r_{0} T^{-1}$.

Theorem 2.1. Let $T \in L(X)$ be an invertible operator whose spectrum contains $\partial \Omega$ such that $T$ and $r_{0} T^{-1}$ are polynomially bounded. Suppose that for some $\varepsilon>0$ and $k \geq 1$, the set

$$
\Lambda_{\varepsilon, k}:=\left\{\lambda \in \Omega: \forall \tilde{\varepsilon}>\varepsilon \exists u \in X \text { with }\|u\|=1 \text { and }\|T u-\lambda u\|<\tilde{\varepsilon}(\operatorname{dist}(\lambda, \partial \Omega))^{k}\right\}
$$

is not an Apostol set. Then T has nontrivial hyper-invariant subspaces.

Lemma 2.2. Let $T \in L(X)$ be an invertible operator such that $T$ and $r_{0} T^{-1}$ are polynomially bounded. Then $\sigma(T) \subset \bar{\Omega}$, and there exists a constant $K$ such that $\|f(T)\| \leq K\|f\|_{A(\bar{\Omega})}$ for all rational functions $f$ whose poles are outside $\bar{\Omega}$. In other words, $\bar{\Omega}$ is a $K$-spectral set for $T$.

Proof. Since both $T$ and $r_{0} T^{-1}$ are polynomially bounded, we have $\sigma(T) \subset \overline{\mathbb{D}}$ and $\sigma\left(r_{0} T^{-1}\right) \subset \overline{\mathbb{D}}$; thus $\sigma(T) \subset \bar{\Omega}$. If $f$ is a rational function with poles outside $\bar{\Omega}$, it can be expressed as the sum of two rational functions $f_{1}, f_{2}$ with $\left\|f_{1}\right\|_{\overline{\mathbb{D}}} \leq$ $c\|f\|_{A(\bar{\Omega})},\left\|f_{2}\right\|_{\mathbb{C} \backslash r_{0} \overline{\mathbb{D}}} \leq c\|f\|_{A(\bar{\Omega})}$ for some constant $c$ (Proposition 9.4 in $[\mathrm{P}]$; the proof is also outlined in Lemma 2.4 below). The lemma follows since $f_{1}$ is a uniform limit of polynomials, $f_{2}$ is a uniform limit of polynomials in $r_{0} z^{-1}$, and $T$ and $r_{0} T^{-1}$ are polynomially bounded.

Since every function in $A(\bar{\Omega})$ can be approximated uniformly by rational functions whose poles are outside $\bar{\Omega}$, we can extend the functional calculus $f \rightarrow f(T)$ to the entire algebra $A(\bar{\Omega})$, and the resulting map will satisfy the inequality $\|f(T)\| \leq$ $K\|f\|_{A(\bar{\Omega})}$ for $f \in A(\bar{\Omega})$. Moreover, we can often extend this functional calculus to $H^{\infty}(\Omega)$ by using the corresponding result of Apostol in the case of the unit disk [A2].

Note that if neither $T^{n} \rightarrow 0$ nor $T^{* n} \rightarrow 0$ in the strong operator topology, then $T^{*}$ has hyper-invariant subspaces by Proposition 3.2 in [A2]; thus, we may assume that at least one of the sequences $\left(T^{n}\right)_{n=1}^{\infty},\left(T^{* n}\right)_{n=1}^{\infty}$ converges to 0 strongly. Under this assumption, Apostol extends the functional calculus from $A(\overline{\mathbb{D}})$ to $H^{\infty}(\mathbb{D})$. This 
extended functional calculus satisfies $h(T) x=\lim _{r \rightarrow 1} h(r T) x$ in the weak topology of $X$. Moreover, $\|h(T)\| \leq c_{T}\|h\|_{\infty}$, where $c_{T}$ is the polynomial bound of $T$.

Similarly, we may assume that either $\left(r_{0} T^{-1}\right)^{n} \rightarrow 0$ or $\left(r_{0} T^{-1}\right)^{* n} \rightarrow 0$ strongly. Thus, $r_{0} T^{-1}$ also has a functional calculus defined on $H^{\infty}(\mathbb{D})$. Since every $h \in$ $H^{\infty}(\Omega)$ can be written uniquely as $h(z)=h_{1}(z)+h_{2}\left(r_{0} / z\right)$ with $h_{1}, h_{2} \in H^{\infty}(\mathbb{D})$ and $h_{2}(0)=0$, we can define $\Phi_{T}: H^{\infty}(\Omega) \rightarrow L(X)$ by $\Phi_{T}(h)=\Phi_{T}\left(h_{1}\right)+$ $\Phi_{r_{0} T^{-1}}\left(h_{2}\right)$. We have $\left\|h_{1}(T)\right\| \leq c_{T}\left\|h_{1}\right\|_{\infty},\left\|h_{2}(T)\right\| \leq c_{r_{0} T^{-1}}\left\|h_{2}\right\|_{\infty}$. By a similar argument as in Lemma 2.2, it follows that $\|h(T)\| \leq K\|h\|_{\infty}$ for some constant $K$ and for every $h \in H^{\infty}(\Omega)$.

Moreover, we can actually assume that the extended functional calculus is an isometry as is verified by the next two lemmas.

Lemma 2.3. If $\Lambda_{\varepsilon, k}$ is an Apostol set for every $\varepsilon>0$ and $k \geq 1$, then $\|h\|_{\infty} \leq$ $\|h(T)\|$ for $h \in H^{\infty}(\Omega)$.

Proof. Let $\varepsilon>0$ and $k \geq 1$ be arbitrary and let $K$ be as above. For $\lambda \in \Lambda_{\varepsilon, k}$ and $h \in H^{\infty}(\Omega)$, there exists a function $g_{\lambda} \in H^{\infty}(\Omega)$ with $h(z)-h(\lambda)=(z-\lambda) g_{\lambda}(z)$ and $\left\|g_{\lambda}\right\| \leq \frac{2\|h\|}{\operatorname{dist}(\lambda, \partial \Omega)}$. We also note that for $\lambda \in \Lambda_{\varepsilon, k}$ and $\tilde{\varepsilon}>\varepsilon$, there exists $v \in X$ with $\|(T-\lambda I) v\|<\tilde{\varepsilon} d i s t(\lambda, \partial \Omega)^{k}$. Thus,

$$
\begin{aligned}
\|h(T)\| & \geq|h(\lambda)|-\|h(T)-h(\lambda) I\| \\
& \geq|h(\lambda)|-2 \tilde{\varepsilon} K\|h\|_{\infty} \operatorname{dist}(\lambda, \partial \Omega)^{k-1} \\
& \geq\|h\|_{\infty}-2 \tilde{\varepsilon} K\|h\|_{\infty} \operatorname{dist}(\lambda, \partial \Omega)^{k-1} \quad\left(\Lambda_{\varepsilon, k} \text { is an Apostol set }\right) \\
& \geq\left(1-2 \tilde{\varepsilon} K \operatorname{dist}(\lambda, \partial \Omega)^{k-1}\right)\|h\|_{\infty} .
\end{aligned}
$$

Since $\varepsilon$ is arbitrary, we have $\|h\|_{\infty} \leq\|h(T)\|$.

Lemma 2.4. If $T \in L(X)$ is such that $\bar{\Omega}$ is a $K$-spectral set for $T$, then $T$ is similar to an operator $T^{\prime}$ on another Banach space $X^{\prime}$ with the property that $\left\|h\left(T^{\prime}\right)\right\| \leq$ $\|h\|_{\infty}$ for all functions $h$ in $A(\bar{\Omega})$. In other words, $\bar{\Omega}$ is a spectral set for $T^{\prime}$.

Proof. Define a new norm on $X$ by $\|x\|^{\prime}=\sup _{f \in A(\bar{\Omega}),\|f\| \leq 1}\|f(T) x\|$. Clearly, $\|x\| \leq$ $\|x\|^{\prime} \leq K\|x\|$, so that $X^{\prime}=\left(X,\|\cdot\|^{\prime}\right)$ is also a Banach space. Moreover, relative to this norm, $\bar{\Omega}$ is a spectral set for $T$ since for $\|f\|_{A(\bar{\Omega})} \leq 1$ and $x \in X$, we have

$$
\|f(T) x\|^{\prime}=\sup _{g \in A(\bar{\Omega}),\|g\| \leq 1}\|g(T) f(T) x\| \leq \sup _{h \in A(\bar{\Omega}),\|h\| \leq 1}\|h(T) x\|=\|x\|^{\prime} .
$$

In the presence of a weakly continuous $H^{\infty}(\Omega)$ functional calculus we also have $\left\|h\left(T^{\prime}\right)\right\| \leq\|h\|_{\infty}$ for every $h \in H^{\infty}(\Omega)$ when $T^{\prime}$ is defined as in the above proof.

Therefore, when proving the main result we may assume that the functional calculus $\Phi_{T}: A(\bar{\Omega}) \rightarrow L(X)$ that maps $1(z) \equiv z$ to $T$ can be extended to $H^{\infty}(\Omega)$, and the extended functional calculus is isometric. Note that when the functional calculus is isometric we always have $\sigma(T) \supset \partial \Omega$. In what follows, we will denote by $\mathbb{A}_{\Omega}(X)$ the set of bounded linear operators $T$ on $X$ which have an isometric functional calculus from $H^{\infty}(\Omega)$ to $L(X)$ that maps $1(z) \equiv z$ to $T$, and by $\Phi_{T}$ the functional calculus corresponding with the operator $T$. 
The algebra $H^{\infty}(\Omega)$ carries a weak* topology viewed as the dual space of $L^{1}(\Omega) /$ ${ }^{\perp} H^{\infty}(\Omega)$. Since the space $L^{1}(\Omega) /{ }^{\perp} H^{\infty}(\Omega)$ is separable, the Krĕ̌n-Šmulian theorem $[\mathrm{C}]$ allows us to work with sequences in proving the weak ${ }^{*}$-continuity of a functional defined on $H^{\infty}(\Omega)$. In particular, we will be interested in weak*-continuity of the functionals $x \otimes_{T} x^{*}: H^{\infty}(\Omega) \rightarrow \mathbb{C}$ defined by $\left\langle h, x \otimes_{T} x^{*}\right\rangle=\left\langle h(T) x, x^{*}\right\rangle$ for $x \in X$, $x^{*} \in X^{*}$, and $h \in H^{\infty}(\Omega)$. We have $\left\|x \otimes_{T} x^{*}\right\| \leq\|x\|\left\|x^{*}\right\|$; thus the functionals $x \otimes_{T} x^{*}$ are bounded. We will show that if $T^{*}$ has no hyper-invariant subspaces, then $x \otimes_{T} x^{*}$ is weak ${ }^{*}$-continuous for all $x \in X$ and $x^{*} \in X^{*}$.

Lemma 2.5. Let $\left(u_{n}\right)$ be a sequence in $H^{\infty}(\Omega)$, and let $v_{n} \in H^{\infty}(\mathbb{D})$, $w_{n} \in$ $H^{\infty}\left(\mathbb{C} \backslash r_{0} \overline{\mathbb{D}}\right)$ be the unique functions satisfying $u_{n}=v_{n}+w_{n}$ for every $n$. If $\left(u_{n}\right)$ converges to 0 in the weak ${ }^{*}$ topology, so do the sequences $\left(v_{n}\right)$ and $\left(w_{n}\right)$.

Proof. Weak* convergence of $u_{n}$ implies that $\sup _{n}\left\|u_{n}\right\|<\infty$ and $u_{n}(z) \rightarrow 0$ for all $z \in \Omega$. Let $\sup _{n}\left\|u_{n}\right\|=M$. For $z \in \mathbb{D}$ and $\varepsilon>0$ sufficiently small, we have

$$
v_{n}(z)=\frac{1}{2 \pi i} \int_{|\zeta|=1-\varepsilon} \frac{u_{n}(\zeta)}{\zeta-z} d \zeta
$$

Since for every $\zeta$ with $|\zeta|=1-\varepsilon, u_{n}(\zeta) \rightarrow 0$, and $u_{n}$ is uniformly bounded, we have $v_{n}(z) \rightarrow 0$ for all $z \in \mathbb{D}$ by Lebesgue's Dominated Convergence Theorem. We can write $v_{n}=u_{n}-w_{n}$ where

$$
w_{n}(z)=\frac{-1}{2 \pi i} \int_{|\zeta|=r_{0}+\varepsilon} \frac{u_{n}(\zeta)}{\zeta-z} d \zeta
$$

For $|z|>1-\varepsilon$,

$$
\left|v_{n}(z)\right| \leq\left|u_{n}(z)\right|+\left|w_{n}(z)\right| \leq M+\left|w_{n}(z)\right| \leq M+M /\left(1-r_{0}-2 \varepsilon\right) .
$$

So, $\sup _{z \in \mathbb{D}}\left|v_{n}(z)\right| \leq M+M /\left(1-r_{0}\right)$. We conclude that $v_{n} \rightarrow 0$ weak*. Similarly, $w_{n} \rightarrow 0$ weak $^{*}$.

Remark 1. It follows from the lemma that if $x \otimes_{T} x^{*}$ is not weak ${ }^{*}$-continuous for some $x \in X$ and $x^{*} \in X^{*}$, then either $x \otimes_{T} x^{*} \mid H^{\infty}(\mathbb{D})$ is not weak*-continuous or $x \otimes_{T} x^{*} \mid H^{\infty}\left(\mathbb{C} \backslash r_{0} \overline{\mathbb{D}}\right)$ is not weak ${ }^{*}$-continuous.

Proposition 2.6. If $T$ is a polynomially bounded operator such that $T^{*}$ has no hyper-invariant subspaces, then $x \otimes_{T} x^{*} \mid H^{\infty}(\mathbb{D})$ is weak ${ }^{*}$-continuous for all $x \in X$ and $x^{*} \in X^{*}$.

Proof. Let $\left(f_{n}\right)_{n=1}^{\infty} \subset H^{\infty}(\mathbb{D})$ be a sequence that converges to 0 in the weak*topology. Note that by Theorem 3.2 in [A2], either $T^{n} \rightarrow 0$ or $T^{* n} \rightarrow 0$ strongly. Then by Proposition 1.8 in $[\mathrm{A} 2], w^{*}-\lim _{n \rightarrow \infty} \Phi_{T}^{*}\left(f_{n}\right) x^{*}=0$ for all $x^{*} \in X^{*}$ where $\Phi_{T}^{*}$ is the functional calculus defined from $H^{\infty}(\mathbb{D})$ to $L\left(X^{*}\right)$ by $\Phi_{T}^{*}(f)=\left(\Phi_{T}(f)\right)^{*}$ for $f \in H^{\infty}(\mathbb{D})$. In other words, $\lim _{n \rightarrow \infty}\left\langle x, \Phi_{T}^{*}\left(f_{n}\right) x^{*}\right\rangle=\lim _{n \rightarrow \infty}\left\langle f_{n}(T) x, x^{*}\right\rangle=0$ for all $x \in X$.

Proposition 2.7. If $T \in \mathbb{A}_{\Omega}(X)$ is such that $T^{*}$ has no hyper-invariant subspaces, then $x \otimes_{T} x^{*}: H^{\infty}(\Omega) \rightarrow \mathbb{C}$ is weak ${ }^{*}$-continuous for all $x \in X$ and $x^{*} \in X^{*}$.

Proof. This follows immediately from Proposition 2.6 and Remark 1.

Based on the reductions discussed above, it is sufficient to prove the following theorem in order to prove our main result. 
Theorem B. Let $T \in \mathbb{A}_{\Omega}(X)$ be such that the set $\Lambda_{k, \varepsilon}$ is an Apostol set for every $\varepsilon>0$ and $k \geq 1$. Assume the functional $x \otimes_{T} x^{*}: H^{\infty}(\Omega) \rightarrow \mathbb{C}$ is weak ${ }^{*}$-continuous for all $x \in X$ and $x^{*} \in X^{*}$. Then there exists a nontrivial common invariant subspace for $T^{*}$ and $T^{*-1}$.

\section{INTERPOLATION IN AN ANNULUS}

The following definition was given by Ambrozie and Müller [AM]:

Definition. Let $L>0$. A collection $\left\{u_{1}, u_{2}, \ldots, u_{n}\right\}$ of nonzero vectors in a Banach space $X$ is said to be $L$-circled if $\left\|\sum_{j=1}^{n} \beta_{j} u_{j}\right\| \leq L\left\|\sum_{j=1}^{n} \gamma_{j} u_{j}\right\|$ whenever $\left|\beta_{j}\right| \leq$ $\left|\gamma_{j}\right|$ for $j=1,2, \ldots, n$.

Ambrozie and Müller $[\mathrm{AM}]$ provided an improvement of Zenger's theorem [BD, pp. 18-20] as follows:

Theorem 3.1. Consider an L-circled set $\left\{w_{1}, w_{2}, \ldots, w_{n}\right\} \subset X$, positive numbers $\alpha_{1}, \alpha_{2}, \ldots, \alpha_{n}$ with $\sum_{j=1}^{n} \alpha_{j}=1$, and a functional $\varphi \in X^{*}$. Then there exist scalars $s_{1}, s_{2}, \ldots, s_{n}$ and $\psi \in X^{*}$ such that $\|\varphi-\psi\| \leq 1,\left\|\sum_{j=1}^{n} s_{j} w_{j}\right\| \leq L \sqrt{2}$, and $\psi\left(s_{j} w_{j}\right)=\alpha_{j}$ for $j=1,2, \ldots, n$.

For the remainder of this paper we will fix $r_{0}<1$, and set $a=4 / r_{0}$. For each $\lambda=r e^{i \theta} \in \Omega$, we define an interval $I_{\lambda}$ in $\partial \Omega$ by

$$
I_{\lambda}:=\left\{\begin{array}{cc}
\left\{e^{i t}:|t-\theta|<a(1-r)\right\} & \text { if } r \geq \sqrt{r_{0}}, \\
\left\{r_{0} e^{i t}:|t-\theta|<a\left(1-\frac{r_{0}}{r}\right)\right\} & \text { if } r<\sqrt{r_{0}} .
\end{array}\right.
$$

These are the analogues of the intervals used in $[\mathrm{AM}]$ (with 2 in place of $a$ ).

We will say that a finite subset $F$ of $\Omega$ is separated if the intervals $\left\{I_{\lambda}: \lambda \in F\right\}$ are pairwise disjoint.

The following lemma is the analogue of Lemma 4.3 in $[\mathrm{AM}]$ with $3 / 4$ replaced by $\sqrt{r_{0}}$ and is proved the same way.

Lemma 3.2. There is a constant $\Delta>0$ with the following property: If $G$ is a separated subset of $\Omega$ such that $|\lambda|>\sqrt{r_{0}}$ for all $\lambda \in G$, then

$$
\prod_{\lambda \in G \backslash\left\{\lambda_{0}\right\}}\left|\frac{\lambda_{0}-\lambda}{1-\bar{\lambda} \lambda_{0}}\right| \geq \Delta \quad \text { for every } \lambda_{0} \in G .
$$

Lemma 3.3. There is a constant $\delta>0$ with the following property: If $F=F_{1} \cup F_{2}$ is a separated subset of $\Omega$ such that $F_{1} \subset\left\{\lambda \in \Omega:|\lambda|>r_{0}{ }^{0.01}\right\}$ and $F_{2} \subset\{\lambda \in \Omega$ : $\left.|\lambda|<r_{0}{ }^{0.99}\right\}$, then

$$
\prod_{\lambda \in F_{1}}\left|\frac{\mu-\lambda}{1-\bar{\lambda} \mu}\right| \geq \delta \quad \text { for every } \mu \in F_{2} .
$$

Upon observing $\left|\frac{\mu-\lambda}{1-\bar{\lambda} \mu}\right| \geq\left(r_{0}^{0.01}-r_{0}^{0.99}\right) / 2$ for every $\lambda \in F_{1}$ and $\mu \in F_{2}$, Lemma 3.3 is also proved the same way as Lemma 4.3 in [AM].

The following result follows from Carleson's interpolation theorem. The proof is analogous to that of Proposition 4.4 in $[\mathrm{AM}]$. See also [Ca]. 
Proposition 3.4. There exists a constant $b$ with the following property: If $G$ is a separated subset of $\Omega$, such that $|\lambda|>\sqrt{r_{0}}$ for every $\lambda \in G$, then given scalars $\left\{c_{\lambda}\right.$ : $\lambda \in G\}$, there exists $f \in H^{\infty}(\Omega)$ such that $\|f\|_{\infty} \leq b \sup _{\lambda \in G}\left|c_{\lambda}\right|$ and $f(\lambda)=c_{\lambda}$ for $\lambda \in G$.

Note that the constant $b$ depends on the constant $\Delta$ obtained in Lemma 3.2.

In what follows $\delta$ and $b$ will stand for the constants obtained in Lemma 3.3 and Proposition 3.4.

Lemma 3.5. Let $F=F_{1} \cup F_{2}$ be a separated subset of $\Omega$ such that $F_{1} \subset\{\lambda \in \Omega$ : $\left.|\lambda|>r_{0}{ }^{0.01}\right\}$ and $F_{2} \subset\left\{\lambda \in \Omega:|\lambda|<r_{0}{ }^{0.99}\right\}$. Then there exist functions $g_{1}$ and $g_{2}$ in $H^{\infty}(\Omega)$, such that $g_{1}(\lambda)=1-g_{2}(\lambda)=0$ for all $\lambda \in F_{1}, g_{1}(\mu)=1-g_{2}(\mu)=$ 1 for all $\mu \in F_{2}$, and $\left\|g_{i}\right\|_{\infty} \leq b / \delta$ for $i=1,2$.

Proof. Define

$$
B_{1}(z)=\prod_{\lambda \in F_{1}} \frac{z-\lambda}{1-\bar{\lambda} z}
$$

By Lemma 3.3, $|B(\mu)| \geq \delta$ for all $\mu \in F_{2}$. Since the intervals $\left\{I_{r_{0} / \mu}: \mu \in F_{2}\right\}$ are pairwise disjoint and $\left|r_{0} / \mu\right|>\sqrt{r_{0}}$ for every $\mu \in F_{2}$, it follows from Proposition 3.4 that there exists a function $f_{1} \in H^{\infty}(\Omega)$ with $f_{1}\left(r_{0} / \mu\right)=1 / B(\mu)$, and $\left\|f_{1}\right\|_{\infty} \leq$ $b / \delta$. Define $g_{1}(z)=f_{1}\left(r_{0} / z\right) B_{1}(z)$. Then $g_{1}(z)=0$ for $z \in F_{1}, \quad g_{1}(z)=$ 1 for $z \in F_{2}$, and $\left\|g_{1}\right\|_{\infty} \leq b / \delta$. We define the second function by $g_{2}(z)=$ $f_{2}(z) B_{2}(z)$ where

$$
B_{2}(z)=\prod_{\mu \in F_{2}} \frac{\frac{r_{0}}{z}-\frac{r_{0}}{\mu}}{1-\frac{r_{0}}{\mu} \frac{r_{0}}{z}}
$$

and $f_{2}$ is the corresponding function obtained by Proposition 3.4 such that $f_{2}(\lambda)=$ $1 / B_{2}(\lambda)$ for $\lambda \in F_{1}$.

Remark 2. In particular for $\lambda_{0} \in F$ we can find $f \in H^{\infty}(\Omega)$ with $f\left(\lambda_{0}\right)=1$, $f(\lambda)=0$ for $\lambda \in F \backslash\left\{\lambda_{0}\right\}$, and $\|f\|_{\infty} \leq b / \delta$.

Let $m$ denote angular measure on $\partial \Omega$. More precisely, the measure of an interval on $\partial \Omega$ is equal to the measure of the corresponding angle with vertex at 0 .

Lemma 3.6. There exists $\kappa>0$ with the following property: If $F=F_{1} \cup F_{2}$ is a separated subset of $\Omega$ such that $F_{1} \subset\left\{\lambda \in \Omega:|\lambda|>r_{0}{ }^{0.01}\right\}, F_{2} \subset\{\lambda \in \Omega:|\lambda|<$ $\left.r_{0}^{0.99}\right\}$, and $\left\{u_{\lambda}: \lambda \in F\right\} \subset X,\left\{\mu_{\lambda}: \lambda \in F\right\} \subset \mathbb{C}$ satisfy $\left\|u_{\lambda}\right\|=1$,

$$
\left\|(T-\lambda I) u_{\lambda}\right\|<\frac{\delta}{2 b \pi}(\operatorname{dist}(\lambda, \partial \Omega))^{2}, \quad \text { and } \quad\left\|\sum_{\lambda \in F} \mu_{\lambda} u_{\lambda}\right\| \leq 1,
$$

then necessarily $\left|\mu_{\lambda}\right| \leq \kappa$.

Proof. Let $\lambda_{0} \in F$ satisfy $\left|\mu_{\lambda_{0}}\right|=\max _{\lambda \in F}\left|\mu_{\lambda}\right|$. Then by the previous remark, there exists $f \in H^{\infty}(\Omega)$ such that $f\left(\lambda_{0}\right)=1, f(\lambda)=0$ for $\lambda \in F \backslash\left\{\lambda_{0}\right\}$, and $\|f\|_{\infty} \leq b / \delta$. Setting $u=\sum_{\lambda \in F} \mu_{\lambda} u_{\lambda}$, we have $\|f(T) u\| \leq b / \delta\|u\| \leq b / \delta$. Now, for $\lambda \in F$ there exists $g_{\lambda}$ analytic on $\Omega$ with $f(z)-f(\lambda)=g_{\lambda}(z)(z-\lambda)$ and 
$\left\|g_{\lambda}\right\|_{\infty} \leq 2\|f\|_{\infty}(\operatorname{dist}(\lambda, \partial \Omega))^{-1} \leq 2(b / \delta)(\operatorname{dist}(\lambda, \partial \Omega))^{-1}$. We have

$$
\begin{aligned}
b / \delta & \geq\|f(T) u\| \\
& \geq\left(\left\|\sum_{\lambda \in F} f(\lambda) \mu_{\lambda} u_{\lambda}\right\|-\left\|\sum_{\lambda \in F} \mu_{\lambda}(f(\lambda)-f(T)) u_{\lambda}\right\|\right) \\
& \geq\left|\mu_{\lambda_{0}} u_{\lambda_{0}}\right|-\sum_{\lambda \in F}\left|\mu_{\lambda}\right| \| g_{\lambda}(T)(T-\lambda I) u_{\lambda}|| \\
& \geq\left|\mu_{\lambda_{0}}\right|-\left|\mu_{\lambda_{0}}\right|\left[\sum_{\lambda \in F_{1}} \frac{2 b}{\delta(1-|\lambda|)} \frac{\delta}{2 \pi b}(1-|\lambda|)^{2}\right. \\
& \geq\left|\mu_{\lambda_{0}}\right|\left(1-\frac{1}{\pi} \sum_{\lambda \in F_{1}}(1-|\lambda|)-\frac{1}{\pi} \sum_{\lambda \in F_{2}} \frac{2 b}{\delta\left(|\lambda|-r_{0}\right)} \frac{\delta}{2 \pi b}\left(|\lambda|-r_{0}\right)^{2}\right] \\
& \geq\left|\mu_{\lambda_{0}}\right|\left(1-\frac{1}{\pi} \sum_{\lambda \in F_{1}} \frac{m\left(I_{\lambda}\right)}{2 a}-\frac{1}{\pi} \sum_{\lambda \in F_{2}} \frac{m\left(I_{\lambda}\right)}{2 a}\right) \\
& \geq\left|\mu_{\lambda_{0}}\right|\left(1-\frac{1}{2 a \pi} 2 \pi-\frac{1}{2 a \pi} 2 \pi\right) \\
& =\left|\mu_{\lambda_{0}}\right|(1-2 / a) .
\end{aligned}
$$

Taking into account that $a>4$, we conclude that the condition of the lemma is true with $\kappa=\frac{b / \delta}{1-2 / a}$.

As in $[\mathrm{AM}]$ we will show in the next two lemmas that any family of vectors $\left\{u_{\lambda}: \lambda \in F\right\}$ which satisfy the hypothesis of the previous lemma are $4 b L$-circled where $L=5+\frac{b}{\delta}$.

Lemma 3.7. Let $F=F_{1} \cup F_{2}$ be a separated subset of $\Omega$ such that $F_{1} \subset\{\lambda \in \Omega$ : $\left.|\lambda|>r_{0}{ }^{0.01}\right\}, F_{2}=\left\{\lambda \in \Omega:|\lambda|<r_{0}{ }^{0.99}\right\}$, and $\left\{u_{\lambda}: \lambda \in F\right\} \subset X$ satisfy $\left\|u_{\lambda}\right\|=1$, and

$$
\left\|(T-\lambda I) u_{\lambda}\right\|<\frac{\delta}{2 b \pi}(\operatorname{dist}(\lambda, \partial \Omega))^{2}
$$

Then given $\beta_{\lambda} \in \overline{\mathbb{D}}$ with $\left\|\sum_{\lambda \in F} \beta_{\lambda} u_{\lambda}\right\|=1$ we have $\left\|x_{j}\right\| \leq L$ where $x_{j}=$ $\sum_{\lambda \in F_{j}} \beta_{\lambda} u_{\lambda}$, for $j=1,2$.

Proof. By Lemma 3.5, there exists $g \in H^{\infty}(\Omega)$ with $g(\lambda)=1$ for $\lambda \in F_{1}$ and $g(\lambda)=0$ for $\lambda \in F_{2}$ and $\|g\|_{\infty} \leq b / \delta$. Let $x=x_{1}+x_{2}$. We have $\left\|x_{1}\right\| \leq$ 


$$
\begin{aligned}
\left\|g(T) x-x_{1}\right\|+\|g(T) x\| \text { and } & \\
\left\|g(T) x-x_{1}\right\| & =\left\|\sum_{\lambda \in F} \beta_{\lambda} g(T) u_{\lambda}-\sum_{\lambda \in F_{1}} \beta_{\lambda} u_{\lambda}\right\| \\
& =\left\|\sum_{\lambda \in F} \beta_{\lambda} g(\lambda) u_{\lambda}+\sum_{\lambda \in F} \beta_{\lambda}(g(T)-g(\lambda)) u_{\lambda}-\sum_{\lambda \in F_{1}} \beta_{\lambda} u_{\lambda}\right\| \\
& =\left\|\sum_{\lambda \in F} \beta_{\lambda}(g(T)-g(\lambda)) u_{\lambda}\right\| .
\end{aligned}
$$

There exists $q_{\lambda} \in H^{\infty}(\Omega)$ with $\left\|q_{\lambda}\right\|_{\infty} \leq 2(\operatorname{dist}(\lambda, \partial \Omega))^{-1}\|g\|_{\infty}$ and $g(z)-g(\lambda)=$ $q_{\lambda}(z)(z-\lambda)$. Thus,

$$
\begin{aligned}
\left\|x_{1}\right\| & \leq \sum_{\lambda \in F} \frac{\delta}{2 b \pi}(\operatorname{dist}(\lambda, \partial \Omega))^{2} 2(\operatorname{dist}(\lambda, \partial \Omega))^{-1}\|g\|_{\infty}+\frac{b}{\delta}\|x\| \\
& =\frac{1}{\pi} \sum_{\lambda \in F} \operatorname{dist}(\lambda, \partial \Omega)+\frac{b}{\delta} \\
& <\frac{1}{\pi} \sum_{\lambda \in F} m\left(I_{\lambda}\right)+\frac{b}{\delta} .
\end{aligned}
$$

Since the intervals $\left\{I_{\lambda}: \lambda \in F\right\}$ are pairwise disjoint, we have $\sum_{\lambda \in F} m\left(I_{\lambda}\right)<4 \pi$. Then, $\left\|x_{1}\right\| \leq 4+\frac{b}{\delta}<L$. We also have $\left\|x_{2}\right\| \leq\left\|x_{1}\right\|+\left\|x_{1}+x_{2}\right\| \leq L$.

Lemma 3.8. Let $F=F_{1} \cup F_{2}$ be a separated subset of $\Omega$ such that $F_{1} \subset\{\lambda \in \Omega$ : $\left.|\lambda|>r_{0}{ }^{0.01}\right\}, F_{2}=\left\{\lambda \in \Omega:|\lambda|<r_{0}{ }^{0.99}\right\}$, and $\left\{u_{\lambda}: \lambda \in F\right\} \subset X$ satisfy $\left\|u_{\lambda}\right\|=1$, and

$$
\left\|(T-\lambda I) u_{\lambda}\right\|<\frac{\delta}{2 b \pi}(\operatorname{dist}(\lambda, \partial \Omega))^{2} .
$$

Then the family $\left\{u_{\lambda}: \lambda \in F\right\}$ is $4 b L$-circled.

Proof. By a trivial modification of Proposition 6.2 of $[\mathrm{AM}]$, we know that $\left\{u_{\lambda}\right.$ : $\left.\lambda \in F_{1}\right\}$ and $\left\{u_{\lambda}: \lambda \in F_{2}\right\}$ are $2 b$-circled. Assume without loss of generality that $\left|\beta_{\lambda}\right| \leq\left|\gamma_{\lambda}\right| \leq 1$ and $\left\|\sum_{\lambda \in F} u_{\lambda} \gamma_{\lambda}\right\|=1$. Then

$$
\begin{aligned}
\left\|\sum_{\lambda \in F} u_{\lambda} \beta_{\lambda}\right\| & \leq\left\|\sum_{\lambda \in F_{1}} u_{\lambda} \beta_{\lambda}\right\|+\left\|\sum_{\lambda \in F_{2}} u_{\lambda} \beta_{\lambda}\right\| \\
& \leq 2 b\left(\left\|\sum_{\lambda \in F_{1}} u_{\lambda} \gamma_{\lambda}\right\|+\left\|\sum_{\lambda \in F_{2}} u_{\lambda} \gamma_{\lambda}\right\|\right) \\
& \leq 4 b L .
\end{aligned}
$$

\section{Poisson KeRnels}

Let $L^{1}(\partial \Omega)$ denote the Banach space of all complex integrable functions in $\partial \Omega$ with respect to $m$ (see Section 3), with

$$
\|f\|_{1}=\int_{\partial \Omega}|f(\zeta)| d m(\zeta) .
$$


Recall that for every $\lambda \in \Omega$ there exists a unique measure $m_{\lambda}$ on $\partial \Omega$ such that

$$
u(\lambda)=\int_{\partial \Omega} u(\zeta) d m_{\lambda}(\zeta)
$$

for every continuous function $u$ in $\bar{\Omega}$ which is harmonic in $\Omega$. The measures $m_{\lambda}$ are absolutely continuous relative to $m$, and the densities $K_{\lambda}=d m_{\lambda} / d m$ have been explicitly evaluated by Sarason [S]. We describe now how the functions $K_{\lambda}$ are obtained.

Define $\widehat{\Omega}=\left\{(r, t): r_{0}<r<1,-\infty<t<\infty\right\}$ so that $\widehat{\Omega}$ is the universal covering surface of $\Omega$ with covering map $\varphi: \widehat{\Omega} \rightarrow \Omega$ defined by $\varphi(r, t)=r e^{i t}$. Define $K$ on $\widehat{\Omega}$ by

where $q_{0}=-\log r_{0}$.

$$
K(r, t)=\frac{\frac{1}{2 q_{0}} \cos \left(\frac{\pi}{q_{0}} \log \frac{r}{\sqrt{r_{0}}}\right)}{\cosh \frac{\pi t}{q_{0}}-\sin \left(\frac{\pi}{q_{0}} \log \frac{r}{\sqrt{r_{0}}}\right)}
$$

The function $K$ enjoys the following properties:

(1) $K(r, t)>0$ for all $(r, t) \in \widehat{\Omega}$.

(2)

$$
\int_{-\infty}^{\infty} K(r, t) d t+\int_{-\infty}^{\infty} K\left(\frac{r_{0}}{r}, t\right) d t=1
$$

(3)

for $r_{0}<r<1$

$$
u\left(r e^{i t}\right)=\int_{-\infty}^{\infty} u\left(e^{i s}\right) K(r, t-s) d s+\int_{-\infty}^{\infty} u\left(r_{0} e^{i s}\right) K\left(\frac{r_{0}}{r}, t-s\right) d s
$$

for all functions $u$ harmonic in $\widehat{\Omega}$.

A calculation shows that

$$
\int K(r, t) d t=\frac{1}{\pi} \arctan \frac{e^{\frac{\pi}{q_{0}} t}-\sin \left(\frac{\pi}{q_{0}} \log \frac{r}{\sqrt{r_{0}}}\right)}{\cos \left(\frac{\pi}{q_{0}} \log \frac{r}{\sqrt{r_{0}}}\right)}+C .
$$

This can be used to calculate the two integrals in (2) separately:

$$
\begin{gathered}
\int_{-\infty}^{\infty} K(r, t) d t=\frac{1}{2}-\frac{\log \frac{r}{\sqrt{r_{0}}}}{\log r_{0}}, \\
\int_{-\infty}^{\infty} K\left(\frac{r_{0}}{r}, t\right) d t=\frac{1}{2}+\frac{\log \frac{r}{\sqrt{r_{0}}}}{\log r_{0}} .
\end{gathered}
$$

Observe also that

$$
\begin{array}{r}
\max _{t} K(r, t)=K(r, 0)=\frac{\frac{1}{2 q_{0}} \cos \left(\frac{\pi}{q_{0}} \log \frac{r}{\sqrt{r_{0}}}\right)}{1-\sin \left(\frac{\pi}{q_{0}} \log \frac{r}{\sqrt{r_{0}}}\right)}, \\
\max _{t} K\left(\frac{r_{0}}{r}, t\right)=K\left(\frac{r_{0}}{r}, 0\right)=\frac{\frac{1}{2 q_{0}} \cos \left(\frac{\pi}{q_{0}} \log \frac{\sqrt{r_{0}}}{r}\right)}{1-\sin \left(\frac{\pi}{q_{0}} \log \frac{\sqrt{r_{0}}}{r}\right)} .
\end{array}
$$


Now for $\lambda=r e^{i \theta} \in \Omega$ we set

$$
K\left(r e^{i \theta}\right):=\sum_{-\infty}^{\infty} K(r, \theta+2 k \pi) .
$$

We then define $K_{\lambda}: \partial \Omega \rightarrow \mathbb{R}$ by $K_{\lambda}\left(e^{i t}\right)=K\left(r e^{i(\theta-t)}\right)$ and $K_{\lambda}\left(r_{0} e^{i t}\right)=$ $K\left(\frac{r_{0}}{r} e^{i(\theta-t)}\right), \quad t \in(-\pi, \pi]$.

Let us recall that $a=4 / r_{0}$. The following is the analogue of Lemma 5.1 in $[\mathrm{AM}]$.

Lemma 4.1. Assume that $\lambda=r e^{i \theta} \in \Omega$ and $r>r_{0}{ }^{0.01}$. Then

$$
\int_{-a(1-r)}^{a(1-r)} K_{\lambda}\left(e^{i(t-\theta)}\right) d t>0.77 \text {. }
$$

Similarly, if $r<r_{0}^{0.99}$,

$$
\int_{-a(1-r)}^{a(1-r)} K_{\lambda}\left(r_{0} e^{i(t-\theta)}\right) d t>0.77 .
$$

Proof. Without loss of generality assume $\lambda=r$.

$$
\begin{aligned}
\int_{-a(1-r)}^{a(1-r)} K_{\lambda}\left(e^{i t}\right) d t & =\int_{-a(1-r)}^{a(1-r)} \sum_{-\infty}^{\infty} K(r,-t+2 k \pi) d t \\
& >\int_{a(r-1)}^{a(1-r)} K(r, t) d t \\
& =2 \int_{0}^{a(1-r)} K(r, t) d t \\
& =\frac{2}{\pi}\left[\arctan \left(\frac{e^{a \frac{\pi}{q_{0}}(1-r)}-\sin \left(\frac{\pi}{q_{0}} \log \frac{r}{\sqrt{r_{0}}}\right)}{\cos }\left(\frac{\pi}{q_{0}} \log \frac{r}{\sqrt{r_{0}}}\right)\right)\right. \\
& \left.-\arctan \left(\frac{1-\sin \left(\frac{\pi}{q_{0}} \log \frac{r}{\sqrt{r_{0}}}\right)}{\cos \left(\frac{\pi}{q_{0}} \log \frac{r}{\sqrt{r_{0}}}\right)}\right)\right]
\end{aligned}
$$

Let $f(r):=\frac{\pi}{q_{0}} \log \left(\frac{r}{\sqrt{r_{0}}}\right), g(r):=\frac{\pi a}{q_{0}}(1-r)$. Note that for $r_{0}<r<1$, we have $-\pi / 2<f(r)<\pi / 2$ and $\cos f(r)>0$. We claim that

$$
\frac{e^{g(r)}-\sin f(r)}{\cos f(r)}>4 \text {. }
$$

We can write

$$
\frac{e^{g(r)}-\sin f(r)}{\cos f(r)}=\frac{e^{g(r)}-1}{\cos f(r)}+\frac{1-\sin f(r)}{\cos f(r)} .
$$

Since

$$
\frac{1-\sin f(r)}{\cos f(r)}>0,
$$

it is enough to prove

$$
\frac{e^{g(r)}-1}{\cos f(r)}>4
$$

in other words, $e^{g(r)}-1-4 \cos f(r)>0$. 
Note that $e^{g(r)}-1-4 \cos f(r)$ is decreasing and converges to 0 as $r \rightarrow 1$. So we have $e^{g(r)}-1-4 \cos f(r)>0$, which in turn implies that

$$
\arctan \left[\frac{e^{g(r)}-\sin f(r)}{\cos f(r)}\right]>\arctan 4>1.32 .
$$

We also observe that

$$
h(r):=\frac{1-\sin f(r)}{\cos f(r)}
$$

is a decreasing function. Now if $r>r_{0}^{0.01}$, then

$$
\begin{aligned}
& -\arctan h(r)>-\arctan \left[\frac{1-\sin \left(\frac{\pi}{-\log r_{0}} \log \frac{r_{0}^{0.01}}{r_{0}{ }^{0.5}}\right)}{\cos \left(\frac{\pi}{-\log r_{0}} \log \frac{r_{0} 0.01}{r_{0}{ }^{0.5}}\right)}\right] \\
& =-\arctan \left[\frac{1-\sin 0.49 \pi}{\cos 0.49 \pi}\right]>-0.016 .
\end{aligned}
$$

Thus

$$
\int_{-a(1-r)}^{a(1-r)} K_{\lambda}\left(e^{i t}\right) d t>\frac{2}{\pi}(1.32-0.016)>0.77,
$$

as claimed. The second result follows immediately as $\left|r_{0} / \lambda\right|>r_{0}^{0.01}$.

We now define functions $Q_{\lambda}: \partial \Omega \rightarrow[0, \infty)$ analogous to the ones defined in $[\mathrm{AM}]$ :

$$
\begin{aligned}
Q_{\lambda}\left(e^{i t}\right) & := \begin{cases}K_{\lambda}\left(e^{i t}\right) & \text { if } e^{i t} \in I_{\lambda}, \\
0 & \text { otherwise, }\end{cases} \\
Q_{\lambda}\left(r_{0} e^{i t}\right) & := \begin{cases}K_{\lambda}\left(r_{0} e^{i t}\right) & \text { if } r_{0} e^{i t} \in I_{\lambda}, \\
0 & \text { otherwise. }\end{cases}
\end{aligned}
$$

Corollary 4.2. For $\lambda=r e^{i \theta}$ with $r>r_{0}^{0.01}$ or $r<r_{0}^{0.99}$,

$$
\int_{\partial \Omega}\left(K_{\lambda}(\zeta)-Q_{\lambda}(\zeta)\right) d m(\zeta) \leq \frac{23}{77} \int_{\partial \Omega} Q_{\lambda}(\zeta) d m(\zeta) .
$$

Proof. Without loss of generality we can assume $r>r_{0}^{0.01}$. Then

$$
\frac{\int_{\partial \Omega}\left(K_{\lambda}(\zeta)-Q_{\lambda}(\zeta)\right) d m(\zeta)}{\int_{\partial \Omega} Q_{\lambda}(\zeta) d m(\zeta)}=\frac{\int_{\partial \Omega} K_{\lambda}(\zeta) d m(\zeta)}{\int_{\partial \Omega} Q_{\lambda}(\zeta) d m(\zeta)}-1 \leq \frac{23}{77}
$$

We define positive numbers $\left\{\gamma_{\lambda}: \lambda \in \Omega\right\}$ by $\gamma_{\lambda}:=\left(\max _{\zeta \in \partial \Omega} K_{\lambda}(\zeta)\right)^{-1}$. The reader will verify without difficulty the following result:

Lemma 4.3. There exists a constant $S>1$ such that

for all $\lambda \in \Omega$.

$$
\frac{m\left(I_{\lambda}\right)}{2 a S} \leq \gamma_{\lambda} \leq \frac{S m\left(I_{\lambda}\right)}{2 a}
$$

In our argument in Section 5 we will need an inner function defined in $\Omega$.

Proposition 4.4. There exists a function u holomorphic on $\bar{\Omega}$ such that $|u(z)|<1$ for $z \in \Omega,|u(z)|=1$ and $u^{\prime}(z) \neq 0$ for $z \in \partial \Omega$. 
Proof. Since $\mathbb{D}$ and $\widehat{\Omega}$ are conformally equivalent, $\mathbb{D}$ constitutes a covering space of $\Omega$ as well. As described in detail in [S], pages 16-20, one can define Blaschke products $H_{a}$ on $\widehat{\Omega}$ for each point $a$ in $\Omega$, by first defining a Blaschke product in $\mathbb{D}$ with simple zeros at each point above $a$ and then transferring it to $\widehat{\Omega}$ via the conformal map between $\mathbb{D}$ and $\widehat{\Omega}$. This leads to the following definition:

For $a \in \Omega, h_{a}\left(r e^{i t}\right):=r^{-\alpha} e^{-i \alpha t} H_{a}(r, t)$ where

$$
\alpha=\left\{\begin{array}{cl}
\frac{1}{q_{0}} \log |a| & \text { for } r_{0}{ }^{1 / 2} \leq|a|<1, \\
\frac{1}{q_{0}} \log \left(|a| / r_{0}\right) & \text { for } r_{0}<|a|<r_{0}{ }^{1 / 2},
\end{array}\right.
$$

which is congruent modulo 1 to the index of $H_{a}$. These functions $h_{a}$ are holomorphic in $\bar{\Omega}$, have modulus one on $C_{1}$, modulus $r_{0}^{-\alpha}$ on $C_{0}$, and $\left|h_{a}(z)\right|<1$ in $\Omega$. For $a=r_{0}^{1 / 2}$ we observe that $H_{a}^{2}$ has index 0 , from which it follows that the function $u$ defined by $u\left(r e^{i t}\right):=H_{a}^{2}(r, t)$ is well-defined and holomorphic in $\bar{\Omega}$, of constant modulus 1 on $\partial \Omega$, and $|u(z)|<1$ in $\Omega$. We now claim that $u^{\prime}(z) \neq 0$ on $\partial \Omega$. In other words the derivative of $H_{a}^{2}(r, t)$ does not vanish on $\partial \widehat{\Omega}$. Since $\widehat{\Omega}, \mathbb{D}$, and $\{z \in \mathbb{C}$ : $\operatorname{Im}(z)>0\}$ are conformally equivalent, and $H_{a}^{2}$ can be extended analytically to $\partial \widehat{\Omega}$, it is enough to show that the derivative of a function $v: \mathbb{C}^{+} \rightarrow \mathbb{C}^{+}$does not vanish on any interval $(a, b)$ in $\mathbb{R}$ where $v:=H_{a}^{2} \circ \phi$ for a biholomorphic map $\phi: \mathbb{C}^{+} \rightarrow \widehat{\Omega}$. A calculation shows that $v^{\prime}(t) \geq 0$ for all $t \in(a, b)$. If $v^{\prime}\left(t_{0}\right)=0$ for some $t_{0}$ in $(a, b)$ such that $v \mid(a, b)$ is real valued, we can write $v(z)-v\left(t_{0}\right)=\left(z-t_{0}\right)^{n} g(z)$ where $g$ is holomorphic with $g\left(t_{0}\right) \neq 0$ and $n \geq 2$. Then $v$ would have to map some points in $\mathbb{C}^{+}$to $\mathbb{C}^{-}$, which contradicts the definition of $v$. Therefore, $u^{\prime}$ does not vanish on $\partial \Omega$.

Note that the Ahlfors function associated with $\Omega$ and $p \in \Omega$ would also satisfy the desired properties. See $[\mathrm{F}]$ for a more detailed discussion of the Ahlfors function.

In the next result,the function $u$ of the preceding proposition plays the role of the function $u(\lambda)=\lambda$ in the arguments of [AM]. Let us fix a constant $N>0$ such that $1 / N \leq\left|u^{\prime}(z)\right| \leq N$ for all $z \in \partial \Omega$.

Lemma 4.5. Let $\Lambda \subset \Omega$ be an Apostol set and $I \subset \partial \Omega$ be an open interval such that $u$ is one-to-one on $I$. Then for sufficiently large $n \in \mathbb{N}$, there exists a separated subset $F$ of $\Lambda$ with the following properties:

(1) $I_{\lambda} \subset I$ for all $\lambda \in F$,

(2) $\left|u(\lambda)^{n}-1\right|<1 / 9$, for all $\lambda \in F$,

(3) $m\left(\bigcup_{\lambda \in F} I_{\lambda}\right) \geq \frac{r_{0}}{40 \pi N^{2}} m(I)$,

(4) $\sum_{\lambda \in F} \gamma_{\lambda} \leq \frac{S}{2 a} m(I)$,

(5) $\int_{\partial \Omega}\left|\sum_{\lambda \in F} \gamma_{\lambda} u(\lambda)^{n} K_{\lambda}(\zeta)-\chi_{I}(\zeta)\right| d m(\zeta) \leq c_{1} m(I)$

where $c_{1}=1-\frac{409 r_{0}}{72,000 a S N^{2} \pi}$.

Proof. Since $u$ is one-to-one on $I$ and $1 / N \leq\left|u^{\prime}(z)\right| \leq N$ for $z \in \partial \Omega,(1 / N) r_{0} m(J)$ $\leq m(u(J))=\int_{J}\left|u^{\prime}(z)\right||d z| \leq N m(J)$ on any subinterval $J$ of $I$. For all $n \geq 1$ set $P_{n}=\left\{z \in u(I):\left|z^{n}-1\right| \leq 1 / 10\right\}$. For $n$ sufficiently large, $m\left(P_{n}\right)>\frac{m(u(I))}{20 \pi}$. Fix such an $n$. Let $R_{n}=\left\{\lambda \in I: u(\lambda) \in P_{n}\right\}$. We have $m\left(R_{n}\right) \geq(1 / N) m\left(P_{n}\right)>$ $\frac{1}{20 \pi N} m(u(I))>\frac{r_{0}}{20 \pi N^{2}} m(I)$. Let $\delta>0$ be such that $m\left(R_{n}\right)-\delta>\frac{r_{0} m(I)}{20 \pi N^{2}}$. Let $B \subset \partial \Omega$ be the set that consists of all points $\zeta \in \partial \Omega$ such that for the argument of $\zeta$, which we denote by $t_{\zeta}$, either $\sup \left\{r \in\left(r_{0}, 1\right): r e^{i t_{\zeta}} \in \Lambda\right\}=1$ or $\inf \left\{r \in\left(r_{0}, 1\right)\right.$ : 
$\left.r e^{i t_{\zeta}} \in \Lambda\right\}=r_{0}$ does not hold. Since $B$ contains at most countably many elements, it can be covered by a countable union $U$ of open intervals with $m(U)<\delta / 2$. Then the set $R^{\prime}=\left(R_{n} \cap \overline{I_{\delta}}\right) \backslash U$, where $\overline{I_{\delta}}$ is a closed interval contained in $I$ with $m\left(I \backslash \overline{I_{\delta}}\right)<\delta / 2$, is compact and $m\left(R^{\prime}\right)>\frac{r_{0} m(I)}{20 \pi N^{2}}$.

Since $\Lambda$ is an Apostol set, and $u$ is continuous, for all $\zeta \in R^{\prime}$ we can find $r_{\zeta}>r_{0}^{0.01}$ or $r_{\zeta}<r_{0}^{0.99}$ such that $\lambda_{\zeta}=r_{\zeta} e^{i t_{\zeta}} \in \Lambda$ and $\left|u\left(\lambda_{\zeta}\right)^{n}-1\right|<1 / 9$ with $I_{\lambda_{\zeta}} \subset I$.

Note that by definition, each $I_{\lambda_{\zeta}}$ is contained entirely either in $C_{0}$ or in $C_{1}$. As $R^{\prime}$ is a compact subset of $\bigcup_{\zeta \in R^{\prime}} I_{\lambda_{\zeta}}$, we can find a finite subcover of $\left(I_{\lambda_{\zeta}}\right)_{\zeta \in R^{\prime}}$ such that any three of the intervals on the same circle have empty intersection. By separating these into two pairwise disjoint families of intervals, and choosing the one with the larger measure, we can find a finite set $F$ such that the intervals $\left(I_{\lambda}\right)_{\lambda \in F}$ are disjoint, and $m\left(\bigcup_{\lambda \in F} I_{\lambda}\right)>m\left(R^{\prime}\right) / 2>r_{0} m(I) /\left(40 N^{2} \pi\right)$.

Part 4 of the lemma follows immediately as a consequence of Lemma 4.3. Indeed,

$$
\sum_{\lambda \in F} \gamma_{\lambda} \leq \frac{S}{2 a} \sum_{\lambda \in F} m\left(I_{\lambda}\right)<\frac{S}{2 a} m(I)
$$

To conclude the proof, observe that

$$
\int_{\partial \Omega}\left|\sum_{\lambda \in F} \gamma_{\lambda} u(\lambda)^{n} K_{\lambda}(\zeta)-\chi_{I}(\zeta)\right| d m(\zeta) \leq I_{1}+I_{2}+I_{3}
$$

where

$$
\begin{aligned}
I_{1} & =\int_{\partial \Omega}\left|\sum_{\lambda \in F} \gamma_{\lambda} u(\lambda)^{n}\left(K_{\lambda}(\zeta)-Q_{\lambda}(\zeta)\right)\right| d m(\zeta) \\
I_{2} & =\int_{\partial \Omega} \sum_{\lambda \in F} \gamma_{\lambda}\left|u(\lambda)^{n}-1\right| Q_{\lambda}(\zeta) d m(\zeta) \\
I_{3} & =\int_{I}\left(1-\sum_{\lambda \in F} \gamma_{\lambda} Q_{\lambda}(\zeta)\right) d m(\zeta)
\end{aligned}
$$

for which we have the following estimates:

$$
\begin{aligned}
I_{1} & \leq \sum_{\lambda \in F} \gamma_{\lambda} \int_{\partial \Omega}\left(K_{\lambda}(\zeta)-Q_{\lambda}(\zeta)\right) d m(\zeta) \\
& \leq \sum_{\lambda \in F} \gamma_{\lambda} \frac{23}{77} \int_{\partial \Omega} Q_{\lambda}(\zeta) d m(\zeta), \\
I_{2} & \leq \frac{1}{9} \sum_{\lambda \in F} \gamma_{\lambda} \int_{I} Q_{\lambda}(\zeta) d m(\zeta), \text { and } \\
I_{3} & =m(I)-\int_{I} \sum_{\lambda \in F} \gamma_{\lambda} Q_{\lambda}(\zeta) d m(\zeta) .
\end{aligned}
$$

Thus,

$$
\int_{\partial \Omega}\left|\sum_{\lambda \in F} \gamma_{\lambda} u(\lambda)^{n} K_{\lambda}(\zeta)-\chi_{I}(\zeta)\right| d m(\zeta) \leq m(I)-\frac{409}{693} \sum_{\lambda \in F} \gamma_{\lambda} \int_{\partial \Omega} Q_{\lambda}(\zeta) d m(\zeta)
$$


It follows from Lemma 4.1 and Lemma 4.3 that

$$
\sum_{\lambda \in F} \gamma_{\lambda} \int_{\partial \Omega} Q_{\lambda}(\zeta) d m(\zeta) \geq \frac{77}{200 S a} \sum_{\lambda \in F} m\left(I_{\lambda}\right) \geq \frac{77}{200 S a} m(I) \frac{r_{0}}{40 N^{2} \pi},
$$

which implies

$$
\int_{\partial \Omega}\left|\sum_{\lambda \in F} \gamma_{\lambda} u(\lambda)^{n} K_{\lambda}(\zeta)-\chi_{I}(\zeta)\right| d m(\zeta) \leq\left(1-\frac{409 r_{0}}{72,000 a S N^{2} \pi}\right) m(I),
$$

as desired.

Fix $c_{2} \in\left(c_{1}, 1\right)$.

Theorem 4.6. Let $f: \partial \Omega \rightarrow \mathbb{C}$ be a nonnegative integrable function and $\Lambda$ be an Apostol set. Then for sufficiently large $n$, there exist a separated subset $F$ of $\Lambda$ and positive numbers $\alpha_{\lambda}(\lambda \in F)$ such that:

(1) $\left|u(\lambda)^{n}-1\right|<1 / 9$ for $\lambda \in F$,

(2) $\sum_{\lambda \in F} \alpha_{\lambda} \leq(S / a)\|f\|_{1}$, and

(3) $\int_{\partial \Omega}\left|\sum_{\lambda \in F} \alpha_{\lambda} u(\lambda)^{n} K_{\lambda}(\zeta)-f(\zeta)\right| d m(\zeta) \leq c_{2}\|f\|_{1}$.

Proof. We may assume that $f \neq 0$. Let $\varepsilon>0$ be such that $\varepsilon<\left(c_{2}-c_{1}\right) / 2$. Let $g$ be a step function such that

$$
\int_{\partial \Omega}|f-g| d m(\zeta) \leq \varepsilon\|f\|_{1} .
$$

Let us write $g=\sum_{j=1}^{n} \beta_{j} \chi_{I_{j}}$ where each $I_{j} \subset \partial \Omega$ is an interval on which $u$ is oneto-one and $\|g\|_{1}=\sum_{j=1}^{n} \beta_{j} m\left(I_{j}\right)$. Applying the previous lemma on each interval $I_{j}$, we obtain finite sets $F_{j}$ and positive numbers $\left\{\gamma_{\lambda}: \lambda \in F_{j}\right\}$ such that either $|\lambda| \geq r_{0}^{0.01}$ or $|\lambda| \leq r_{0}^{0.99}$ for $\lambda \in F$,

$$
\sum_{\lambda \in F_{j}} \gamma_{\lambda} \leq \frac{S}{2 a} m\left(I_{j}\right)
$$

and

$$
\int_{\partial \Omega}\left|\sum_{\lambda \in F_{j}} \gamma_{\lambda} u(\lambda)^{n} K_{\lambda}(\zeta)-\chi_{I_{j}}(\zeta)\right| d m(\zeta) \leq c_{1} m\left(I_{j}\right)
$$

Let $F=\bigcup_{j=1}^{n} F_{j}$ and $\alpha_{\lambda}=\beta_{j} \gamma_{\lambda} \quad$ for $\quad \lambda \in F_{j}$. Note that $F_{j}$ can be taken to be pairwise disjoint. Then

$$
\begin{aligned}
& \sum_{\lambda \in F} \alpha_{\lambda} \leq \frac{S}{2 a} \int_{\partial \Omega} g(\zeta) d m(\zeta) \leq \frac{S}{2 a}\left(\int_{\partial \Omega} f(\zeta) d m(\zeta)+\int_{\partial \Omega}|f(\zeta)-g(\zeta)| d m(\zeta)\right) \\
& \quad \leq \frac{S}{2 a}(1+\varepsilon) \int_{\partial \Omega} f(\zeta) d m(\zeta) \leq\|f\|_{1} \frac{S}{a} .
\end{aligned}
$$

To conclude the proof it will be enough to show that

$$
\int_{\partial \Omega}\left|\sum_{\lambda \in F} \alpha_{\lambda} u(\lambda)^{n} K_{\lambda}(\zeta)-g(\zeta)\right| d m(\zeta) \leq\left(c_{1}+\varepsilon\right)\|f\|_{1} .
$$


Indeed,

$$
\begin{aligned}
& \int_{\partial \Omega}\left|\sum_{\lambda \in F} \alpha_{\lambda} u(\lambda)^{n} K_{\lambda}(\zeta)-g(\zeta)\right| d m(\zeta) \\
\leq & \sum_{j=1}^{n} \beta_{j} \int_{\partial \Omega}\left|\sum_{\lambda \in F_{j}}\left(\gamma_{\lambda} u(\lambda)^{n} K_{\lambda}(\zeta)-\chi_{I_{j}}(\zeta)\right)\right| d m(\zeta) \\
\leq & \sum_{j=1}^{n} \beta_{j} c_{1} m\left(I_{j}\right) \\
= & c_{1}\|g\|_{1},
\end{aligned}
$$

and the desired inequality follows since $\|g\|_{1} \leq\|f\|_{1}+\|g-f\|_{1}<(1+\varepsilon)\|f\|_{1}$.

\section{MAin RESUlt}

For a further reduction of the proof of Theorem A, we consider the sets $M=\{x$ : $\left.u(T)^{n} x \rightarrow 0\right\}$ and $M_{*}=\left\{x^{*}: u(T)^{* n} x \rightarrow 0\right\}$. The assumption that $T \in \mathbb{A}_{\Omega}(X)$ implies that $u(T)$ is power bounded. It is also easy to verify that $\sigma(u(T))$ contains the unit circle. Then by Theorem 3.2 in [A2], if neither $M=X$ nor $M_{*}=X^{*}$, it follows that $u(T)^{*}$ has hyper-invariant subspaces, and therefore so does $T^{*}$. Thus, to obtain the main result it is enough to prove the following theorem.

Theorem C. Let $T \in \mathbb{A}_{\Omega}(X)$ be such that the set $\Lambda_{k, \varepsilon}$ is an Apostol set for every $\varepsilon>0$ and $k \geq 1$. Assume that the functional $x \otimes_{T} x^{*}: H^{\infty}(\Omega) \rightarrow \mathbb{C}$ is weak ${ }_{-}$ continuous for all $x \in X$ and $x^{*} \in X^{*}$, and $u(T)^{n} x \rightarrow 0$ for all $x \in X$. Then there exists a nontrivial common invariant subspace for $T$ and $T^{-1}$.

In the remainder of the section, we will prove Theorem C. For $f \in L^{1}(\partial \Omega)$ we will denote by $M_{f}$ the functional defined by

$$
M_{f}(h)=\int_{\partial \Omega} f(\zeta) h(\zeta) d m(\zeta) \quad \text { for } \quad h \in A(\bar{\Omega}) .
$$

In particular, we will denote by $E_{\lambda}$ the functionals corresponding to Poisson kernels $K_{\lambda}$ defined in Section 4 . We have $\left\|M_{f}\right\| \leq\|f\|_{1}$ for all $f \in L^{1}(\partial \Omega)$ and

$$
E_{\lambda}(h)=\int_{\partial \Omega} K_{\lambda}(\zeta) h(\zeta) d m(\zeta)=h(\lambda), \quad h \in A(\bar{\Omega}) .
$$

The hypothesis of Theorem $\mathrm{C}$ implies that for given $x \in X, x^{*} \in X^{*}$, there exists $f \in L^{1}(\partial \Omega)$ such that

$$
\left\langle h(T) x, x^{*}\right\rangle=\int_{-\pi}^{\pi} h\left(e^{i t}\right) f\left(e^{i t}\right) d t+\int_{-\pi}^{\pi} h\left(r_{0} e^{i t}\right) f\left(r_{0} e^{i t}\right) d t, \quad h \in A(\bar{\Omega}) .
$$

Our goal is to show that for every $g \in L^{1}(\partial \Omega)$, there exist $x \in X, x^{*} \in X^{*}$ such that $M_{g}(h)=\left(x \otimes_{T} x^{*}\right)(h)$ for all $h \in A(\bar{\Omega})$.

Fix a constant $c_{3} \in\left(c_{2}, 1\right)$.

Proposition 5.1. Assume that the hypothesis of Theorem $\mathrm{C}$ is satisfied. Fix a nonnegative function $f \in L^{1}(\partial \Omega)$ with $\|f\|_{1}=1$ and $y^{*} \in X^{*}$. Then for $n$ sufficiently large, there exist $x \in X$ and $x^{*} \in X^{*}$ such that $\|x\| \leq 4 \sqrt{2} S b L / a,\left\|x^{*}\right\| \leq$ 1 and $\left\|x \otimes_{T}\left(u(T)^{* n} x^{*}+y^{*}\right)-M_{f}\right\|<c_{3}$. 
Proof. Let $\varepsilon>0$ be such that $\varepsilon<1 / 2, \varepsilon<1 /\left\|y^{*}\right\|^{2}, \varepsilon<\delta /(4 b \pi)$, and $64 \kappa \pi S b L \sqrt{\varepsilon}$ $<c_{3}-c_{2}$ where $\kappa$ is the constant obtained in Lemma 3.6. Denote by $\Lambda$ the collection of all points $\lambda \in \Omega$ such that $|\lambda|<r_{0}^{0.99}$ or $|\lambda|>r_{0}^{0.01}$ and for all $\tilde{\varepsilon}>\varepsilon$ there exists $w \in X$ with $\|w\|=1,\|(T-\lambda I) w\|<\tilde{\varepsilon}(\operatorname{dist}(\lambda, \partial \Omega))^{2}$. By assumption, $\Lambda$ is an Apostol set. Fix $\varepsilon<\tilde{\varepsilon}<2 \varepsilon$. Let $n \in \mathbb{N}$ be large enough. Choose a set $F \subset \Lambda$ and constants $\left\{\alpha_{\lambda}: \lambda \in F\right\}$ satisfying conditions (1)-(3) of Theorem 4.6, and define

$$
g(\zeta):=\sum_{\lambda \in F} \alpha_{\lambda} u(\lambda)^{n} K_{\lambda}(\zeta)
$$

We have then

$$
\begin{aligned}
& M_{g}(h)=\sum_{\lambda \in F} \alpha_{\lambda} u(\lambda)^{n} h(\lambda), \quad h \in A(\bar{\Omega}), \quad \text { and } \\
& \left\|M_{g}-M_{f}\right\| \leq\|f-g\|_{1} \leq c_{2}\|f\|_{1} .
\end{aligned}
$$

For each $\lambda \in F$, fix $w_{\lambda} \in X$ with $\left\|w_{\lambda}\right\|=1$ such that $\left\|(T-\lambda I) w_{\lambda}\right\|<$ $\varepsilon(\operatorname{dist}(\lambda, \partial \Omega))^{2}$. Define on the linear span of $\left\{w_{\lambda}: \lambda \in F\right\}$, a linear functional $\varphi$ by $\varphi\left(w_{\lambda}\right)=u(\lambda)^{-n}\left\langle w_{\lambda}, y^{*}\right\rangle$. By the Hahn-Banach theorem, we can extend this to a functional, still denoted $\varphi$, on $X$. We know by Lemma 3.8 that these $w_{\lambda}$ are $4 b L$-circled. Using the generalization Theorem 3.1 of Zenger's theorem, we find complex numbers $\mu_{\lambda}$ and a functional $\psi \in X^{*}$ such that $\|\psi-\varphi\| \leq 1,\left\|\sum_{\lambda \in F} \mu_{\lambda} w_{\lambda}\right\| \leq$ $4 \sqrt{2} S b L / a$, and $\psi\left(\mu_{\lambda} w_{\lambda}\right)=\alpha_{\lambda}$ for all $\lambda \in F$. Note that $\left|\mu_{\lambda}\right| \leq 4 \sqrt{2} \kappa S b L / a$ by Lemma 3.6.

We claim that $x=\sum_{\lambda \in F} \mu_{\lambda} w_{\lambda}$ and $x^{*}=\psi-\varphi$ satisfy the conditions of our proposition. Clearly, $\|x\| \leq 4 \sqrt{2} S b L / a$ and $\left\|x^{*}\right\| \leq 1$, so it remains to show that $\left\|x \otimes_{T}\left(u(T)^{* n} x^{*}+y^{*}\right)-M_{f}\right\|<c_{3}$. As $\left\|M_{g}-M_{f}\right\| \leq\|g-f\|_{1} \leq c_{2}$, it suffices to show that $\left\|x \otimes_{T}\left(u(T)^{* n} x^{*}+y^{*}\right)-M_{g}\right\|<c_{3}-c_{2}$. Observe that

$$
\begin{aligned}
\| x & \otimes_{T}\left(u(T)^{* n} x^{*}+y^{*}\right)-M_{g} \| \\
& =\left\|\sum_{\lambda \in F} \mu_{\lambda} w_{\lambda} \otimes_{T} u(T)^{* n} x^{*}+\sum_{\lambda \in F} \mu_{\lambda} w_{\lambda} \otimes_{T} y^{*}-M_{g}\right\| \\
& =\sup _{\|h\| \leq 1, h \in A(\bar{\Omega})}\left|\sum_{\lambda \in F}\left(\mu_{\lambda} w_{\lambda} \otimes_{T} u(T)^{* n} x^{*}\right)(h)+\sum_{\lambda \in F}\left(\mu_{\lambda} w_{\lambda} \otimes_{T} y^{*}\right)(h)-M_{g}(h)\right| . \\
(\star) & =\sup _{\|h\| \leq 1, h \in A(\bar{\Omega})}\left|\sum_{\lambda \in F}\left\langle\mu_{\lambda} h(T) w_{\lambda}, u(T)^{* n} x^{*}\right\rangle+\sum_{\lambda \in F}\left\langle\mu_{\lambda} h(T) w_{\lambda}, y^{*}\right\rangle-M_{g}(h)\right| .
\end{aligned}
$$

The first sum in the last line above is equal to

$$
\sum_{\lambda \in F}\left\langle\mu_{\lambda}\left(u(T)^{n} h(T)-u(\lambda)^{n} h(\lambda)\right) w_{\lambda}, x^{*}\right\rangle+\sum_{\lambda \in F}\left\langle\mu_{\lambda} u(\lambda)^{n} h(\lambda) w_{\lambda}, x^{*}\right\rangle,
$$

while the second is equal to

$$
\sum_{\lambda \in F} \mu_{\lambda}\left\langle(h(T)-h(\lambda)) w_{\lambda}, y^{*}\right\rangle+\sum_{\lambda \in F}\left\langle\mu_{\lambda} h(\lambda) w_{\lambda}, y^{*}\right\rangle .
$$


Since $x^{*}=\psi-\varphi, y^{*}\left(w_{\lambda}\right)=u(\lambda)^{n} \varphi\left(w_{\lambda}\right)$, and $\psi\left(\mu_{\lambda} w_{\lambda}\right)=\alpha_{\lambda}$, we have

(3) $\sum_{\lambda \in F}\left\langle\mu_{\lambda} u(\lambda)^{n} h(\lambda) w_{\lambda}, x^{*}\right\rangle+\sum_{\lambda \in F}\left\langle\mu_{\lambda} h(\lambda) w_{\lambda}, y^{*}\right\rangle=\sum_{\lambda \in F} \alpha_{\lambda} u(\lambda)^{n} h(\lambda)=M_{g}(h)$.

Using equations $(1),(2),(3)$, the last line in $(\star)$ can be written as

$$
\begin{array}{r}
\sup _{\|h\| \leq 1, h \in A(\bar{\Omega})} \mid \sum_{\lambda \in F}\left\langle\mu_{\lambda}\left(u(T)^{n} h(T)-u(\lambda)^{n} h(\lambda)\right) w_{\lambda}, x^{*}\right\rangle \\
+\sum_{\lambda \in F}\left\langle\mu_{\lambda}(h(T)-h(\lambda)) w_{\lambda}, y^{*}\right\rangle \mid .
\end{array}
$$

For each $\lambda \in F$ and $h \in A(\bar{\Omega})$ with $\|h\|_{A(\bar{\Omega})}=1$, there exists $q_{\lambda}^{h} \in A(\bar{\Omega})$ with $u(z)^{n} h(z)-u(\lambda)^{n} h(\lambda)=(z-\lambda) q_{\lambda}^{h}(z)$ and $\left\|q_{\lambda}^{h}\right\| \leq 2(\operatorname{dist}(\lambda, \partial \Omega))^{-1} ; p_{\lambda}^{h} \in A(\bar{\Omega})$ with $h(z)-h(\lambda)=(z-\lambda) p_{\lambda}^{h}(z)$ and $\left\|p_{\lambda}^{h}\right\| \leq 2(\operatorname{dist}(\lambda, \partial \Omega))^{-1}$.

So we can rewrite $(\star)$ as

$$
\sup _{\|h\| \leq 1, h \in A(\bar{\Omega})}\left|\sum_{\lambda \in F}\left\langle\mu_{\lambda}(T-\lambda I) q_{\lambda}^{h}(T) w_{\lambda}, x^{*}\right\rangle+\sum_{\lambda \in F}\left\langle\mu_{\lambda}(T-\lambda I) p_{\lambda}^{h}(T) w_{\lambda}, y^{*}\right\rangle\right| .
$$

Then, finally,

$$
\begin{aligned}
(\star) & \leq(8 \sqrt{2} \kappa S b L / a)(\tilde{\varepsilon}+\sqrt{\tilde{\varepsilon}}) \sum_{\lambda \in F} \operatorname{dist}(\lambda, \partial \Omega) \\
& \leq(16 \sqrt{2} \kappa S b L \sqrt{\tilde{\varepsilon}} / a) \sum_{\lambda \in F} \frac{m\left(I_{\lambda}\right)}{2 a} \\
& \leq 64 \kappa \pi S b L \sqrt{\varepsilon} \\
& <c_{3}-c_{2} .
\end{aligned}
$$

From now on $\eta$ will denote the constant $4 \sqrt{2} S b L / a$.

Lemma 5.2. Assume that the hypothesis of Theorem $\mathrm{C}$ is satisfied. Then for given $y \in X, y^{*} \in X^{*}, \varepsilon>0$, and a nonnegative function $f \in L^{1}(\partial \Omega)$, there exist $w \in X$ and $w^{*} \in X^{*}$ such that

(1) $\left\|w \otimes_{T}\left(w^{*}+y^{*}\right)-M_{f}\right\| \leq c_{3}\|f\|_{1}$,

(2) $\left\|y \otimes_{T} w^{*}\right\|<\varepsilon$,

(3) $\|w\| \leq \eta \sqrt{\|f\|_{1}}$ and $\left\|w^{*}\right\| \leq \sqrt{\|f\|_{1}}$.

Proof. Choose $n$ so that $\left\|u(T)^{n} y\right\| \leq \frac{\varepsilon}{\|f\|_{1}^{1 / 2}}$. By applying the previous proposition to the function $f /\|f\|_{1}$, and to the functional $y^{*} / \sqrt{\|f\|_{1}}$, we get

$$
\left\|v \otimes_{T}\left(u(T)^{* n} v^{*}+\frac{y^{*}}{\|f\|^{1 / 2}}\right)-M_{f /\|f\|_{1}}\right\|<c_{3}
$$

for some $v$ and $v^{*}$ with $\|v\| \leq \eta$ and $\left\|v^{*}\right\| \leq 1$. Let $w=v\|f\|_{1}^{1 / 2}$ and $w^{*}=$ $\|f\|_{1}^{1 / 2} u(T)^{* n} v^{*}$. 
Then

$$
\begin{gathered}
\left\|w \otimes_{T}\left(w^{*}+y^{*}\right)-M_{f}\right\|<c_{3}\|f\|_{1}, \\
\left\|y \otimes_{T} w^{*}\right\|=\left\|y \otimes_{T} \sqrt{\|f\|_{1}} u(T)^{* n} v^{*}\right\|=\left\|u(T)^{n} y \otimes_{T} \sqrt{\|f\|_{1}} v^{*}\right\| \\
\leq\left\|u(T)^{n} y\right\|\left\|v^{*}\right\|\|f\|_{1}^{1 / 2}<\varepsilon \\
\|w\| \leq \eta \sqrt{\|f\|_{1}} \text {, and }\left\|w^{*}\right\| \leq \sqrt{\|f\|_{1}} .
\end{gathered}
$$

Fix an integer $N$ such that $c_{3}+\pi N^{-1}<1$, and a positive constant $c$ satisfying $1-N^{-1}\left(1-c_{3}-\pi N^{-1}\right)<c<1$.

Lemma 5.3. Assume that the hypothesis of Theorem $\mathrm{C}$ is satisfied. Then for given $y \in X, y^{*} \in X^{*}, h \in L^{1}(\partial \Omega)$, there exist $x \in X$ and $x^{*} \in X^{*}$ such that

(1) $\|y-x\| \leq \eta\|h\|_{1}^{1 / 2}$,

(2) $\left\|y^{*}-x^{*}\right\| \leq \sqrt{\|h\|_{1}}$,

(3) $\left\|x \otimes_{T} x^{*}-y \otimes_{T} y^{*}-M_{h}\right\| \leq c\|h\|_{1}$.

Proof. Without loss of generality, we may assume that $\|h\|_{1} \neq 0$. For $j=0,1, \ldots$, $N-1$, let $B_{j}$ be the set of all complex numbers that are of the form $r e^{i t}$ with $r>0$ and $-\pi / N \leq t-2 \pi j / N<\pi / N$. Fix a representative of $h$ and define $A_{j}=h^{-1}\left(B_{j}\right)$ for $j=0,1,2, \ldots, N-1$. Then $\|h\|_{1}=\sum_{j=0}^{N-1}\left\|h \chi_{A_{j}}\right\|_{1}$. Fix $0 \leq j_{0} \leq N-1$ such that $\left\|h \chi_{A_{j_{0}}}\right\|_{1} \geq N^{-1}\|h\|_{1}$, and set $v=e^{2 \pi j_{0} i / N}$. For each $\zeta \in A_{j_{0}}$, we have

$$
|v| h(\zeta)|-h(\zeta)|=|h(\zeta)|\left|v-\frac{h(\zeta)}{|h(\zeta)|}\right| \leq|h(\zeta)| \pi N^{-1}
$$

So

$$
\left\|v|h| \chi_{A_{j_{0}}}-h \chi_{A_{j_{0}}}\right\|_{1} \leq \pi N^{-1}\left\|h \chi_{A_{j_{0}}}\right\|_{1}
$$

Choose $\varepsilon>0$ such that $\varepsilon\|h\|_{1}^{-1}+1-N^{-1}\left(1-c_{3}-\pi N^{-1}\right)<c$. By Lemma 5.3, there exist vectors $w \in X$ and $w^{*} \in X^{*}$ such that $\|w\| \leq \eta\left\|h \chi_{A_{j_{0}}}\right\|_{1}^{1 / 2},\left\|w^{*}\right\| \leq\left\|h \chi_{A_{j_{0}}}^{1 / 2}\right\|_{1}$, $\left\|y \otimes_{T} w^{*}\right\|<\varepsilon$, and

$$
\left\|w \otimes_{T}\left(w^{*}+y^{*}\right)-M_{|h| \chi_{A_{j_{0}}}}\right\| \leq c_{3}\left\|h \chi_{A_{j_{0}}}\right\|_{1}
$$

We claim that $x=y+v w$ and $x^{*}=y^{*}+w^{*}$ satisfy the desired conditions. Clearly,

$$
\begin{gathered}
\|x-y\|=\|v w\| \leq \eta\left\|h \chi_{A_{j_{0}}}\right\|_{1}^{1 / 2} \leq \eta\|h\|_{1}^{1 / 2}, \\
\left\|y^{*}-x^{*}\right\|=\left\|w^{*}\right\| \leq\|h\|_{1}^{1 / 2} .
\end{gathered}
$$


Furthermore,

$$
\begin{aligned}
& \left\|x \otimes_{T} x^{*}-y \otimes_{T} y^{*}-M_{h}\right\| \leq\left\|y \otimes_{T} w^{*}\right\|+\left\|v w \otimes_{T} x^{*}-M_{h}\right\| \\
& \leq\left\|y \otimes_{T} w^{*}\right\|+\left\|v\left(w \otimes_{T}\left(y^{*}+w^{*}\right)-M_{|h| \chi_{A_{j_{0}}}}\right)\right\| \\
& +\left\|v M_{|h| \chi_{A_{j}}}-M_{h}\right\| \\
& \leq \varepsilon+c_{3}\left\|h \chi_{A_{j_{0}}}\right\|_{1}+\left\|v|h| \chi_{A_{j_{0}}}-h \chi_{A_{j_{0}}}\right\|_{1} \\
& +\sum_{j \neq j_{0}}\left\|h \chi_{A_{j_{0}}}\right\|_{1} \\
& \leq \varepsilon+\left(c_{3}+\pi N^{-1}\right)\left\|h \chi_{A_{j_{0}}}\right\|_{1}+\|h\|_{1}-\left\|h \chi_{A_{j_{0}}}\right\|_{1} \\
& \leq \varepsilon+\|h\|_{1}-\left\|h \chi_{A_{j_{0}}}\right\|_{1}\left(1-c_{3}-\pi N^{-1}\right) \\
& \leq \varepsilon+\|h\|_{1}\left(1-N^{-1}\left(1-c_{3}-\pi N^{-1}\right) \leq c\|h\|_{1}\right. \text {. }
\end{aligned}
$$

Lemma 5.4. Assume that the hypothesis of Theorem $\mathrm{C}$ is satisfied. Then for all $g \in L^{1}(\partial \Omega)$, there exist $x \in X$ and $x^{*} \in X^{*}$ such that $M_{g}=x \otimes_{T} x^{*}$.

Proof. We will proceed by induction. Set $x_{0}=0, x_{0}^{*}=0$, and $\phi_{0}=x_{0} \otimes x_{0}^{*}-M_{g}$. Then $\left\|\phi_{0}\right\|=\|g\|_{1}$. Suppose we have constructed vectors $x_{j} \in X$ and $x_{j}^{*} \in X^{*}$ such that $\left\|\phi_{j}\right\| \leq c^{j}\|g\|_{1}$ where $\phi_{j}=x_{j} \otimes_{T} x_{j}^{*}-M_{g}$. There exists $h_{j} \in L^{1}(\partial \Omega)$ representing $\phi_{j}$ with $\left\|h_{j}\right\|=\left\|\phi_{j}\right\|$. By the previous lemma, there exist $x_{j+1} \in$ $X, x_{j+1}^{*} \in X^{*}$ such that $\left\|x_{j+1}-x_{j}\right\| \leq \eta\left\|h_{j}\right\|_{1}^{1 / 2} \leq \eta c^{j / 2}\|g\|_{1}^{1 / 2}$ and $\left\|x_{j+1}^{*}-x_{j}^{*}\right\| \leq$ $c^{j / 2}\|g\|_{1}^{1 / 2}$, and moreover we have the estimate $\left\|x_{j+1} \otimes_{T} x_{j+1}^{*}-x_{j} \otimes_{T} x_{j}^{*}+M_{h_{j}}\right\| \leq$ $c\left\|h_{j}\right\|_{1}$. Thus $\left(x_{j}\right)$ and $\left(x_{j}^{*}\right)$ are Cauchy sequences. Let $x=\lim _{j \rightarrow \infty} x_{j}$ and $x^{*}=$ $\lim _{j \rightarrow \infty} x_{j}^{*}$. For all $f \in A(\bar{\Omega})$ with $\|f\|=1$ we have

$$
\begin{gathered}
\left|\left\langle f(T) x_{j}, x_{j}{ }^{*}\right\rangle-\left\langle f(T) x, x^{*}\right\rangle\right| \\
\leq\left|\left\langle f(T) x_{j}, x_{j}^{*}\right\rangle-\left\langle f(T) x_{j}, x^{*}\right\rangle\right|+\left|\left\langle f(T) x_{j}, x^{*}\right\rangle-\left\langle f(T) x, x^{*}\right\rangle\right| \\
\leq\left\|x_{j}^{*}-x^{*}\right\|\left\|x_{j}\right\|+\left\|x_{j}-x\right\|\left\|x^{*}\right\| \rightarrow 0 .
\end{gathered}
$$

On the other hand, by the previous lemma,

$$
\begin{gathered}
\left\|\phi_{j+1}\right\|=\left\|x_{j+1} \otimes_{T} x_{j+1}^{*}-M_{g}\right\|=\left\|x_{j+1} \otimes_{T} x_{j+1}^{*}-x_{j} \otimes_{T} x_{j}^{*}+\phi_{j}\right\| \\
=\left\|x_{j+1} \otimes_{T} x_{j+1}^{*}-x_{j} \otimes_{T} x_{j}^{*}+M_{h_{j}}\right\| \leq c\left\|h_{j}\right\|_{1}<c^{j+1}\|g\|_{1} .
\end{gathered}
$$

Thus, $x \otimes_{T} x^{*}=\lim _{j \rightarrow \infty} x_{j} \otimes_{T} x_{j}^{*}=M_{g}$.

Note that there exists a constant $k$ such that $x$ and $x^{*}$ of the previous lemma can be chosen to satisfy $\|x\| \cdot\left\|x^{*}\right\| \leq k\|g\|_{1}$ where the constant $k$ is independent of $g$.

Proof of Theorem $C$. Fix $\lambda_{0} \in \partial \Omega$. By the previous lemma there exist $x \in X$ and $x^{*} \in X^{*}$ such that $x \otimes_{T} x^{*}=E_{\lambda_{0}}$. Thus $\left\langle f(T) x, x^{*}\right\rangle=f\left(\lambda_{0}\right)$ for all $f \in A(\bar{\Omega})$. We may assume $\left(T-\lambda_{0} I\right) x \neq 0$, since in that case $\operatorname{Ker}\left(T-\lambda_{0} I\right)$ is a hyper-invariant subspace for $T$. Then the closure of $\left\{f(T) x: f \in A(\bar{\Omega}), f\left(\lambda_{0}\right)=0\right\}$ is the desired common invariant subspace for $T$ and $T^{-1}$. 


\section{ACKNOWLEDGMENT}

I would like to express my gratitude to my advisor Hari Bercovici for his invaluable guidance and support. I would also like to thank the referee for his suggestions and corrections.

\section{REFERENCES}

[AM] C. Ambrozie, V. Müller, Invariant subspaces for polynomially bounded operators, J. Functional Analysis, 213 (2004), 321-345. MR2078629 (2005d:47013)

[A1] C. Apostol, Utraweakly closed operator algebras, J. Operator Theory, 2 (1979), 49-61. MR553863 (80k:47005)

[A2] , Functional calculus and invariant subspaces, J. Operator Theory, 4 (1980), 159190. MR595410 (82j:47005)

[B] H. Bercovici, Notes on invariant subspaces, Bull. Amer. Math. Soc., 23 (1990), 1-36. MR990552 (90k:47013)

[BCP] S. Brown, B. Chevreau, C. Pearcy, On the structure of contraction operators. II, J. Functional Analysis, 76 (1988), 30-55. MR923043 (90b:47030b)

[BD] F.F. Bonsall, J. Duncan, Numerical ranges II, Cambridge University Press, Cambridge, 1973. MR0442682 (56:1063)

[C] J. B. Conway, A course in functional analysis, second ed., Springer-Verlag, New York, 1990. MR1070713 (91e:46001)

[Ca] L. Carleson, An interpolation problem for bounded analytic functions, Amer. J. Math., 80 (1958), 921-930. MR0117349 (22:8129)

[F] S.D. Fischer, Function theory on planar domains, John Wiley \& Sons, New York, 1983. MR694693 (85d:30001)

[P] V. I. Paulsen, Completely bounded maps and dilations, Longman Scientific \& Technical, Harlow, 1986. MR868472 (88h:46111)

[S] D. Sarason, The $H^{p}$ spaces of an annulus, Mem. Amer. Math. Soc., 56 (1965). MR0188824 $(32: 6256)$

Department of Mathematics, Indiana University, Rawles Hall, Bloomington, Indiana 47405

E-mail address: oyavuz@indiana.edu

Current address: Department of Mathematics, Middle East Technical University, 06531 Ankara, Turkey

E-mail address: oyavuz@metu.edu.tr 